\title{
The Spiral Interplanetary Magnetic Field: A Polarity and Sunspot Cycle Variation
}

by

Leif Svalgaard and John M. Wilcox

June 1974

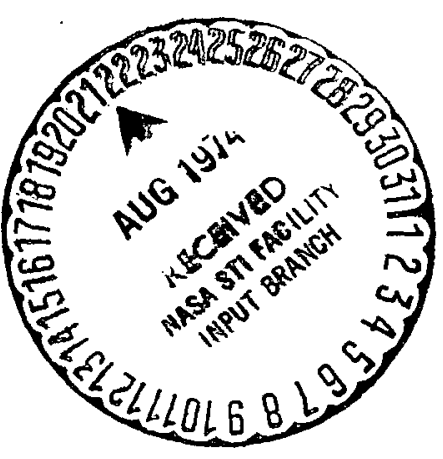

SUIPR Report No. 573

Office of Naval Research

Contract N00014-67-A-0112-0068

National Aeronautics and Space Administration

Grant NGR 05-020-559

and

National Science Foundation

Grant GA-3II38

(NASA-CR-139499) - THE SPIRAL INTERPLANETARY MAGNETIC FIELD: A POLARITY. AND SUNSPOT. CYCLE VARIATION

(Stanford Univ.) : 11 p. HC $\$ 4.00$-CSCL .03B

Depp

\section{INSTITUTE FOR PLASMA RESEARCH STANFORD UNIVERSITY, STANFORD, CALIFORNIA}


THE SPIRAL INTER PLANETARY MAGNETIC FIELD:

A POLARITY AND SUNSPOT CYCLE VARIATION

by

Leif Svalgaard and John M. Wilcox

Office of Naval Research

Contract N00014-67-A-0112-0068

National Aeronautics and Space Administration

Grant NGR 05-020-559

and

National Science Foundation

Grant GA-31138

SUIPR Report No, 573

June 1974

Institute for Plasma Research

Stanford University

Stanford, California

Submitted to Science. 
THE SPIRAL INTERPLANETARY MAGNETIC FIELD:

A POLARITY AND SUNSPOT CYCLE VARIATION

by

Leif Svalgaard and John M. Wilcox

\begin{abstract}
Spacecraft observations near the earth of the yearly average direction of the interplanetary magnetic field during the sunspot maximum year 1968 showed a deviation from the spiral field of E. N. Parker's classical description. The included angle between the average field direction when the field polarity was away from the sun and the average direction for toward polarity was $168^{\circ}$, rather than $180^{\circ}$ as predicted by Parker. This effect appears to have a sunspot cycle variation.
\end{abstract}


The Spiral Interplanetary Magnetic Field:

A Polarity and Sunspot Cycle Variation

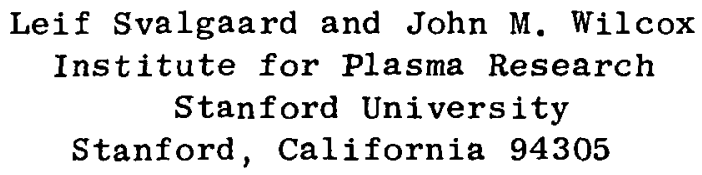

The solar magnetic field is stretched out away from the sun by the solar wind plasma. Parker (1) pointed out that the resulting interplanetary magnetic field should on the average have the form of an Archimedes spiral because of the combined effects of the radially flowing solar wind and a twisting induced by solar rotation. Near the earth the average radial solar wind velocity is approximately $400 \mathrm{~km} / \mathrm{sec}$ which is approximately equal to the azimuthal velocity of solar rotation $\Omega R$, where $\Omega$ is the sun's angular velocity and $R$ is the distance from sun to earth, so that the average spiral angle is about $45^{\circ}$.

Early spacecraft observations (reviewed by Wilcox. (2)), showed that on the average the interplanetary magnetic field configuration was close to that described by Parker, and this theoretical picture is now widely accepted. A recent investigation utilizing the several years of spacecraft observations of the interplanetary magnetic field that are now available has uncovered a variation in the average spiral angle of the field observed near the earth having a dependence on the polarity of the field (away from the sun or toward the sun) and 'on the time within the . eleven year sunspot cycle. This effect may be considered as a perturbation of the basic description of Parker (1).

The effect may be described with reference to Figure 1, in which the earth is at the center of the coordinate system and the direction of 
the earth's orbital velocity is toward the right. The average spiral angle of about $45^{\circ}$ as described by parker is represented by the dashed 1ine. During the sunspot maximum year of 1968 the observed yearly average field directions were qualitatively as shown by the solid lines labeled "AWAY" and "TOWARD." That is, the average spiral field direction was different when the polarity of the interplanetary field was away from the sun than when it was toward the sun. The included angle $\delta$ was about $168^{\circ}$ rather than the expected $180^{\circ}$ : The "AWAY" and "TOWARD" average field directions were symmetrically displaced from the $45^{\circ}$ spiral line, i.e. the average of $\alpha_{A}$ and $\alpha_{T}$ was approximately $45^{\circ}$.

The variation of this effect through a portion of a sunspot cycle is shown in the lower portion of Figure 2 . The angle $\delta^{0}$ represents the yearly average of the angle $\delta$ shown in Figure 1 , and points are plotted for each year. The spacecraft with which the observations were made is indicated by the label in the corner of the figure, and generally a few thousand hours of data go into each average. Note that in the years 1967 and 1968 Explorer 33 and Explorer 35 observed essentially the same value of $\delta^{\circ}$. This tends to reduce the possibility of the observed effect being an artifact, because of the very different orbits and spin directions of the two spacecraft. Explorer 33 was orbiting the earth with its spin axis nearly parallel to the ecliptic plane. Explorer 35 was orbiting the moon with its spin axis nearly perpendicular to the ecliptic plane. The probable error for Explorer 33 observations during 1968 is $\pm 1^{\circ}$, and the probable errors for the other years are comparable.

The upper portion of Figure 2 shows the yearly averages of the 
average spiral angle $\alpha^{\circ}$, which is computed from hourly averages of $\alpha_{\mathrm{A}}$ and $\alpha_{\mathrm{T}}$. The average spiral angle remains within one or two degrees of $45^{\circ}$, thus showing that the effect that we discuss tends to be symmetric about the Parker (1) spiral angle, and also indicating that our numerical analysis has a resulting scatter of only one or two degrees, as compared with a range in the included angle of $\delta^{\circ}$ of almost $20^{\circ}$ as shown in the bottom portion of Figure 2 .

We will discuss possible physical explanations of this effect for the sunspot maximum year 1968; when the amplitude of the effect was the largest. Analogous considerations may be applied to the other years. The most obvious possibility would be that the average solar wind velocity was considerably larger when the interplanetary field was directed away from the sun than when it was toward the sun, since in the Parker (1) description the spiral angle $\alpha$ is given by $\tan \alpha=\Omega R / V_{S}$, where $V_{S}$ is the solar wind velocity. However, to explain the effect in this way during 1968 would have required an extremely large difference in the two solar wind velocities. When the interplanetary field was away from the sun, the average velocity would have to be $530 \mathrm{~km} / \mathrm{sec}$, and when the field was toward the sun the average velocity would have to be 350 $\mathrm{km} / \mathrm{sec}$. During the year July 1967 to July 1968 (the limits are set by spacecraft launch and failure of the instrument) the MIT solar wind plasma experiment on Explorer 35 had a computed yearly average solar velocity of $470 \mathrm{~km} / \mathrm{sec}$ while the field polarity was away from the sun and of 455 $\mathrm{km} / \mathrm{sec}$ while the field polarity was toward the sun. Thus the effect cannot be explained in terms of the average solar wind velocity.

Deflections in the direction of the solar wind velocity at the interface between steady corotating solar wind streams were predicted by 
Carovillano and Siscoe (3) and observed by Ness et al. (4). Such deflections would appear to be averaged out in the yearly averages considered here, and should not contribute to the effect.

If magnetic reconnection were occurring only between a preceding away polarity sector and a following toward polarity sector the effect might be explained. But then $\delta$ would be very different near a boundary in which the field polarity changed from toward to away as compared with near a boundary in which the field polarity changed from away to toward. An analysis computing values of $\delta$ for two-day intervals centered on sector boundaries showed that $\delta$ was the same within two degrees irrespective of the polarity change across the boundary. The possibility of explaining the effect through magnetic reconnection is an attractive one, but it appears that the observations rule out any simple application of this. The effect is formally equivalent to a constant field of magnitude approximately one-fifth the spiral field directed normal to the usual spiral direction. Near the sun this would be equivalent to an azimuthal field that could be produced by an electric current flowing approximately along the sun's rotation axis from the nearby interstellar medium into the southern solar hemisphere and out of the northern solar hemisphere into the interstellar medium. We are not aware of any evidence for such a current, although Schatten and Wilcox (5) have discussed the possibility of magnetic reconnection between the solar polar magnetic field and the nearby interstellar magnetic field. Another observed deviation from the Parker model was reported by Ness et al. (4) who found no trace of the variation in average magnetic 
field direction as a function of velocity that would be expected from the equation $\tan \alpha=\Omega R / V_{S}$. A similar result was found by Neugebauer and Snyder (6). 


\section{References and Notes}

1. E. N. Parker, Astrophys. J. 128, 664 (1958).

2. J. M. Wilcox, Space Sci. Revs. 8, 258 (1968).

3. R. L. Carovillano and G. L. Siscoe, Solar Phys. $\underline{8}, 401$ (1969).

4. N. F. Ness, A. J. Hundhausen and S. J. Bame, J. Geophys. Res. 76 , $6643(1971)$.

5. K. H. Schatten and J. M. Wilcox, J. Geophys. Res. 74, 4157 (1969),

6. M. Neugebauer and C. W. Snyder, J. Geophys. Res. 72, 1823 (1967).

7. Acknowledgments: We thank Peter Hedgecock for the HEOS 1 and 2 observations of the interplanetary magnetic field. We thank Joseph King of the National Space Sciences Data Center for assistance in obtaining the other spacecraft observations. This work was supported in part by the Office of Naval Research under Contract N00014-67-A-0112-0068, by the National Aeronautics and Space Administration under Grant NGR 05-020-559, and by the Atmospheric Sciences Section of the National Science Foundation under Grant GA-31138.

8. May 1974 . 
Figure Captions

\section{Figure 1}

Geometry of the interplanetary magnetic field. The earth is at the center of the coordinate system and its orbital velocity is toward the right. The average Archimedian spiral direction described by Parker (1) is indicated by the dashed line. The average interplanetary field direction when the field polarity is away from the sun is indicated by the arrow labeled"AWAY" with a spiral angle $\alpha_{A}$, and similarly for the field toward the sun. The included angle between the average away direction and the average toward direction is labeled $\delta$. In the classical Parker description $\delta \stackrel{1}{=} 180^{\circ}$.

\section{Figure 2}

Yearly averages $\delta^{\circ}$ of the included angle defined in Figure 1 through a portion of a sunspot cycle. The spacecraft with which each yearly average was obtained is indicated in the box in the corner. The average spiral angle $\alpha^{\circ}$ which is equal to the average of $\alpha_{A}$ and $\alpha_{T}$ is. also shown. 


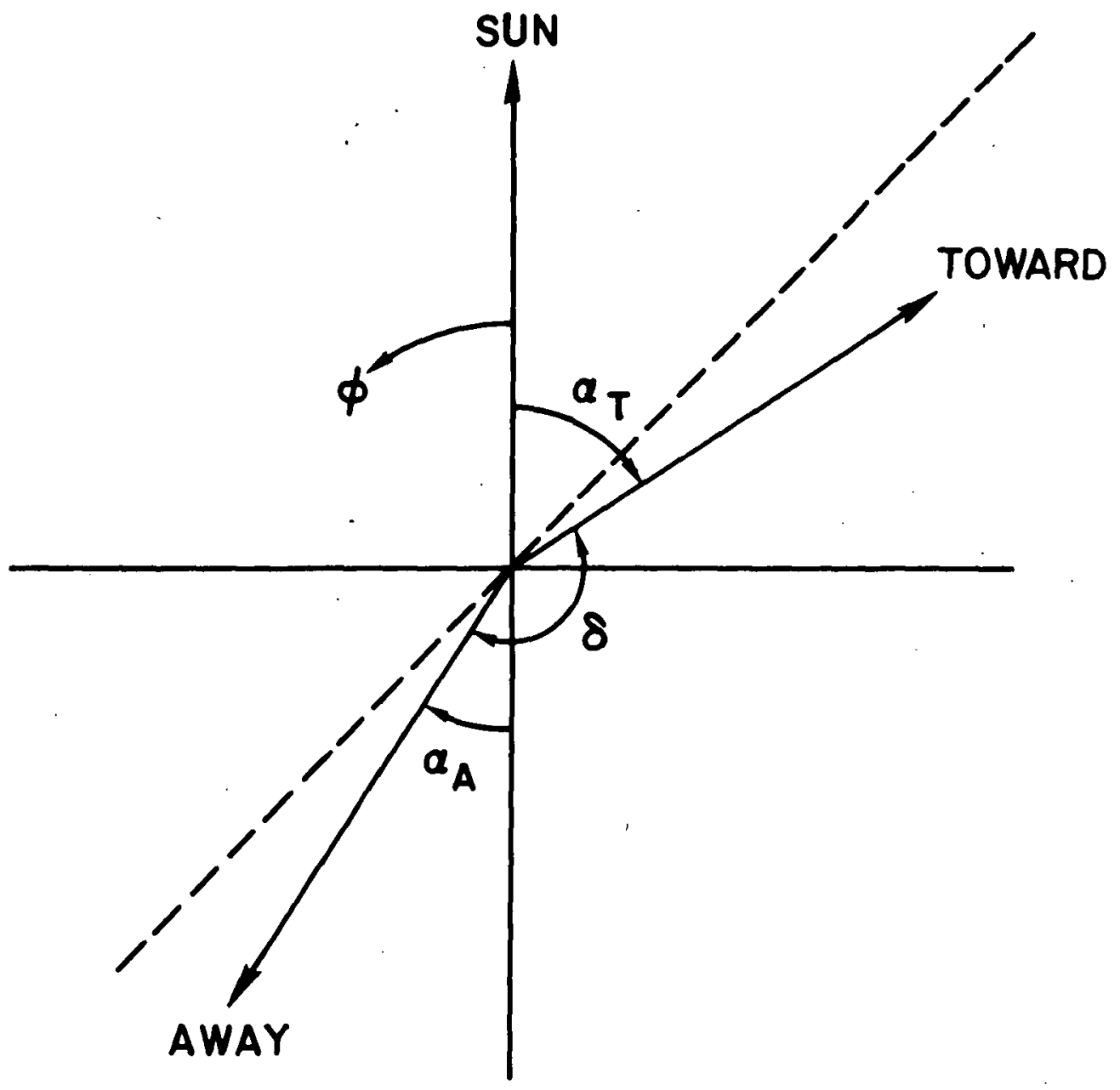

Figure 1 


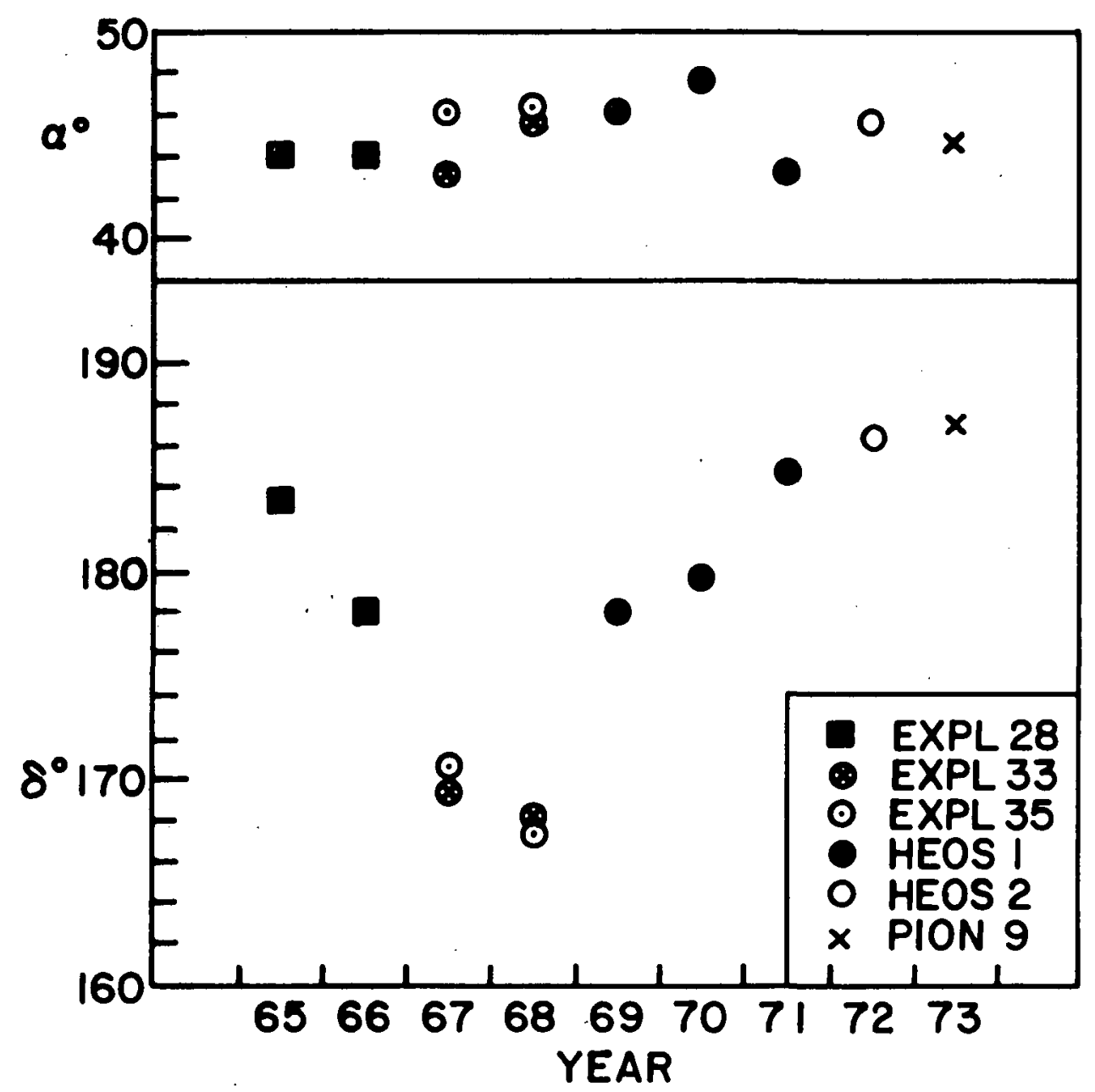

Figure 2 
UNCLASSIF I ED

SECURITY CLASSIFICATION OF THIS PAGE (When Data Entered)

\begin{tabular}{|c|c|}
\hline REPORT DOCUMENTATION PAGE & $\begin{array}{l}\text { READ INSTRUCTIONS } \\
\text { BEFORE COMPLETING FORM }\end{array}$ \\
\hline $\begin{array}{l}\text { 1. REPORT NUMBER } \\
\qquad \text { SUIPR Report No. } 573\end{array}$ & 3. RECIPIENT'S CATALOG NUMBER \\
\hline $\begin{array}{l}\text { 4. TITLE (and Subtitle) } \\
\text { THE SPIRAL INTERPLANETARY MAGNETIC FIELD: } \\
\text { A POLARITY AND SUNSPOT CYCLE VARIATION }\end{array}$ & $\begin{array}{l}\text { 5. TYPE OF REPORT \& PERIOD COVEREO } \\
\text { Scientific } \\
\end{array}$ \\
\hline & 6. PERFORMING ORG. REPORT NUMBER \\
\hline $\begin{array}{l}\text { 7. AUTHOR(s) } \\
\text { Leif Svalgaard and John M. Wilcox }\end{array}$ & $\begin{array}{l}\text { 8. CONTRACT OR GRANT NUMBER(s) } \\
\text { NO0014-67-A-0112-0068 }\end{array}$ \\
\hline $\begin{array}{l}\text { 9. PERFORMING ORGANIZATION NAME AND ADDRESS } \\
\text { Institute for Plasma Research } \\
\text { Stanford University } \\
\text { Via Crespi, Stanford, California } 94305\end{array}$ & $\begin{array}{l}\text { 10. PROGRAM ELEMENT, PROJECT. TASK } \\
\text { AREA \& WORK UNIT NUMBERS }\end{array}$ \\
\hline 11. CONTROLLING OFFICE NAME AND ADDRESS & \begin{tabular}{l|c} 
12. REPORT DATE & 13. NO. OF PACE: \\
June 1974 & 17
\end{tabular} \\
\hline $\begin{array}{l}\text { Office of Naval Research } \\
800 \text { North Quincy street } \\
\text { Arlington, Virginia } 22217\end{array}$ & $\begin{array}{l}\text { 15. SECURITY CLASS. (of this report) } \\
\text { UNCLASSIFIED }\end{array}$ \\
\hline 14. MONITORING AGENCY NAME \& ADDRESS (if diff. from Controlling Office) & $\begin{array}{l}\text { 15a. DECLASSIFICATION/DOWNGRADING } \\
\text { SCHEDULE }\end{array}$ \\
\hline $\begin{array}{l}\text { 16. DISTRIBUTION STATEMENT (of this report) } \\
\text { This document has been approved for public release } \\
\text { is unlimited. }\end{array}$ & nd sale; its distribution \\
\hline 17. DISTRIBUTION STATEMENT (of the abstract entered in Block 20 , if different & om reportl \\
\hline $\begin{array}{l}\text { 18. SUPPL EMENTARY NOTES } \\
\text { TECH; OTHER }\end{array}$ & \\
\hline $\begin{array}{l}\text { 19. KEY WORDS (Continue on reverse side if necessary and identify by block numbe } \\
\text { INTERPLANETARY MAGNETIC FIELD } \\
\text { INTER PLANETARY MAGNETIC SECTOR STRUCTURE } \\
\text { INTERPLANETARY MAGNETIC SPIRAL ANGLE } \\
\text { v }\end{array}$ & \\
\hline $\begin{array}{l}\text { 20. ABSTRACT (Continue on reverse side if necessary and identify by block number) } \\
\text { Spacecraft observations near the earth of the } \\
\text { the interplanetary magnetic field during the sunspo } \\
\text { deviation from the spiral field of E. N. Parker's c } \\
\text { included angle between the average field direction } \\
\text { away from the sun and the average direction for tow } \\
\text { rather than } 180^{\circ} \text { as predicted by Parker. This effe } \\
\text { cycle variation. }\end{array}$ & $\begin{array}{l}\text { early average direction of } \\
\text { maximum year } 1968 \text { showed a } \\
\text { lassical description. The } \\
\text { wen the field polarity was } \\
\text { ard polarity was } 168^{\circ} \text {, } \\
\text { appears to have a sunspot }\end{array}$ \\
\hline
\end{tabular}

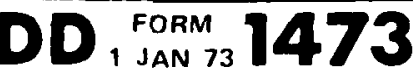

EDITION OF 1 NOV 65 IS OBSOLETE 